\section{Treatment and Outcome of Psychogenic Nonepileptic Seizures}

Ronald P. Lesser, M.D.

\section{Introduction}

The treatment of a disorder typically is based on an understanding of the mechanisms of the disorder and its etiology, permitting a suitable treatment to be directed at the appropriate cause. What happens when the observed symptoms have multiple potential causes? This situation is not unusual in medicine: a cough may be due to a cold or to cancer. Likewise, psychogenic nonepileptic seizures (PNESs) outwardly may appear similar to epilepsy but are caused purely by the emotions. Moreover, diagnosis and treatment may be more difficult because some caregivers are not comfortable diagnosing or treating patients with emotional disorders, such as PNESs.

\section{The Complex Etiology of PNES}

Clearly, more than one possible reason exists for emotional distress, and this fact is as true for patients with PNESs as it is for any other psychiatric disorder. The first step in understanding PNESs is to consider whether certain specific kinds of emotional difficulties underlie certain specific manifestations of PNESs. Broadly, PNESs can present either with prominently motor components or with prominently affective components, and several studies have suggested that these different presentations might differentiate between good and bad outcomes $(1,2)$. For example, one study found that patients with prominent motor components to their PNESs were less likely to achieve seizure control (3). Similarly, a second study demonstrated that patients without rigidity, shaking, ictal incontinence, tongue biting, episodes of PNES status, or admissions to an intensive care unit had better outcomes (4). However, a third study reported that patients with prominently affective components and fewer motor features to their episodes appeared to be more emotionally disturbed (5). The report further suggested that PNES episodes might be more likely to persist in patients with prominently affective components, although the study was not

Epilepsy Currents, Vol. 3, No. 6 (November/December) 2003 pp. 198-200 Blackwell Publishing, Inc.

(c) American Epilepsy Society designed to assess the question. Therefore disagreement is found in the literature: the clinical manifestations of PNESs may help in determining prognosis in some circumstances, but not in others.

As with other types of "conversion reactions," a great many causes for PNESs have been described in the literature (6-8). Little is known about whether discrete conversion symptoms are more likely to have a specific etiology, regardless of the type of symptom. The etiologies can be grouped into several broad categories.

- First, PNES episodes can occur as a result of a disturbance in the patient's interactions with others or in another's interactions with the patient. For instance, some patients with inadequate personalities, adjustment reactions, family conflicts, or who have been victims of sexual or physical abuse are at greater risk of developing physical expressions of their emotional difficulties, such as PNESs. Family, friends, or co-workers may have reinforced a patient's PNES behaviors. Patients who have difficulties managing anger or controlling their hostility toward others may manifest these difficulties with PNESs.

- Second, episodes can be due to intrinsic emotional problems or to internalized conflicts. This set of etiologies includes affective disorders, panic attacks, anxiety, obsessive-compulsive disorders, conversion/somatization or dissociative/depersonalization disorders, and posttraumatic stress disorders. Patients may, for internal reasons, misinterpret or over interpret occurrences in the environment, including patients in whom simple partial seizures may occur and elaborate into PNESs (9).

- Third, PNES episodes occur among patients who have a history of psychosis, such as schizophrenia. In many cases, the type of psychosis is not clearly reported in the published study.

- Fourth, PNES patients may exhibit personality disorders. Possible etiologies in this group include borderline, histrionic, narcissistic, antisocial, passive-aggressive, avoidant, and passive-dependent personality. Etiologies such as malingering, factitious disorder, and substance abuse can be included in this group as well.

- Fifth, cognitive difficulties or a history of head trauma may exist. Some studies report an increased likelihood of 
magnetic resonance imaging (MRI) or electroencephalogram (EEG) abnormalities or frontal lobe impairment among patients with PNESs (10-12).

- Finally, some etiologies, such as attention deficit disorder and tic, are mentioned in the literature but are difficult to classify into the schema outlined previously.

There can be more than one etiology for PNES in a given patient-who you are and how you interact with others are closely intertwined. In addition, in a significant subgroup of patients, a psychiatric diagnosis cannot be made (10,13-16). The personalities of these patients may be variants, even if extreme variants, of normal. Because these individuals can seem so normal otherwise, it can be difficult to understand why PNESs are occurring. However, these patients may fare relatively well clinically, as will be discussed.

\section{Presenting the Diagnosis to the PNES Patient}

Telling the patient the diagnosis is the first step in treatment. This should be done carefully and tactfully. A person with primary responsibility for conducting the evaluation should be the first to tell the patient. This can be complicated once a patient is admitted for video-EEG monitoring. At that point, many caregivers have access to the patient's chart and to the patient. Some clinicians may discuss the diagnosis prematurely with the patient, and the preliminary diagnosis of PNESs, perhaps based on an initial outpatient assessment, may even be inaccurate. Not all caregivers are trained or skilled in treating patients with conversion symptoms and may not inform patients of their diagnoses as tactfully as they should. Therefore it is a good idea to avoid writing in the chart that the patient may have PNESs until the diagnosis is confirmed and the patient has been properly informed.

\section{Exploring Etiology with the Patient}

The next step in treatment of PNESs is to explore with the patient why the events are occurring. Some patients may know why, and their impressions should be discussed. These conversations alone are sufficient to allow control of PNES in some patients: two of nine patients in one series and three of 27 in another $(2,17)$. Nevertheless, most of the patients will need additional evaluation and treatment before they can control their PNESs. Patients often resist recommendations for emotional assessment, making it particularly important to present the diagnosis and recommend treatment in a manner that is sensitive to the patient's sense of personal dignity (18). It also is important to find a therapist who is comfortable assessing and treating patients with conversion symptoms-not all are.

\section{Treatment of the PNES Patient}

Among six studies that included 317 patients, 29\% to 52\% became seizure free, and $15 \%$ to $43 \%$ experienced seizure reduction $(2,13,15,19-21)$. Evidence suggests that the likelihood of long-term seizure control depends more on the presence or absence of an underlying psychiatric diagnosis than it does on the treatment. Lempert and Schmidt (13) compared PNES patients with and without additional psychiatric diagnoses. Of those with psychiatric diagnoses, seven became episode free, and 23 did not. Of those without psychiatric diagnoses, six became seizure free, and two did not. The difference was significant $(P<0.05)$. Similar findings subsequently have been demonstrated in several other studies $(4,6)$.

The treatment(s) given should be tailored to the underlying emotional cause(s) of the PNESs, and treatments will vary depending on the goals that are set (see Table 1).

Among the studies of treatment of PNESs, no single strategy appears most effective $(4,10,14,15,21)$. Both outpatient and inpatient therapy have been used as well as both "talking therapies" and behavioral techniques. Two of these studies found that patients with somatization or dissociative disorders were less likely to respond to treatment $(4,10)$. Although there is an increasing emphasis on medication in psychiatry in general, studies on treatment of PNES generally do not emphasize the use of drugs, and it is not clear how helpful drugs are for this disorder. It is more likely that specific drugs would be useful for specific target symptoms.

Structural problems occur in treating PNES patients because of both staffing and health insurance barriers involved in securing the appropriate clinicians $(14,22)$. Consequently, the treating practitioners may have only limited familiarity and comfort with epilepsy and PNESs. Results are likely to

TABLE 1. Treatment of Psychogenic Nonepileptic Seizures

\begin{tabular}{ll}
\hline Goals & \multicolumn{1}{c}{ Means } \\
\hline Acceptance & Explanation \\
Motivation & Exploration \\
Understanding & Counseling \\
& Psychotherapy \\
Control & Biofeedback \\
& Counseling \\
& Meditation \\
& Medication \\
& Psychotherapy \\
Consolidation & Relaxation therapy \\
& Follow-up \\
\hline
\end{tabular}

The table outlines potential goals for patients with PNES in the left column. The right column outlines potential therapeutic means to meet these goals. 
be better when treating personnel are experienced with both these entities.

Whatever the treatment chosen, an important actor at this stage is the neurologist or epileptologist who made the initial diagnosis and discussed it with the patient. That person should see the patient again to learn whether treatment has been initiated and if it has been effective. When appropriate, the patient should be encouraged to continue in treatment.

\section{Conclusion}

In summary, PNESs vary in their clinical manifestations and vary in their etiologies. No single treatment can be prescribed for all patients with PNES, any more than a single treatment can be prescribed for all patients with epilepsy. Because of their underlying emotional makeup, some patients resist treatment and control of their episodes (23). Other patients are better able to control their episodes and their lives. Patients who are found to be emotionally normal tend to do well, perhaps regardless of the treatment they receive.

We must develop better methods for determining etiologies underlying PNESs in individual patients. Ultimately, improved care of these patients also will depend on increasing their access to practitioners familiar with their condition, skilled in applying the many treatment approaches that have been demonstrated to be effective, and able to select the right treatment for the individual patient.

\section{References}

1. Leis AA, Ross MA, Summers AK. Psychogenic seizures: Ictal characteristics and diagnostic pitfalls. Neurology 1992;42:95-99.

2. Meierkord H, Will B, Fish D, Shorvon S. The clinical features and prognosis of pseudoseizures diagnosed using video-EEG telemetry. Neurology 1991;41:1643-1646.

3. Selwa LM, Geyer J, Nikakhtar N, Brown MB, Schuh LA, Drury I. Nonepileptic seizure outcome varies by type of spell and duration of illness. Epilepsia 2000;41(10):1330-1334.

4. Reuber M, Pukrop R, Bauer J, Helmstaedter C, Tessendorf N, Elger CE. Outcome in psychogenic nonepileptic seizures: 1 to 10 year follow-up in 164 patients. Ann Neurol 2003;53:305-311.

5. Holmes GL, Sackellares JC, McKiernan J, Ragland M, Dreifuss FE. Evaluation of childhood pseudoseizures using EEG telemetry and video tape monitoring. J Pediatr 1980;97:554-558.
6. Lesser RP. Psychogenic seizures. Neurology 1996;46:1499-1507.

7. Gumnit RJ, Gates JR. Psychogenic seizures. Epilepsia 1986;27(suppl 2):S124-S129.

8. Gates JR, Erdahl P. Classification of non-epileptic events. In: Rowan AJ, Gates JR, eds. Non-epileptic seizures. Stoneham, MA: Butterworth-Heinemann, 1993:21-30.

9. Kapur J, Pillai A, Henry TR. Psychogenic elaboration of simple partial seizures. Epilepsia 1995;36(11):1126-1130.

10. Kanner AM, Parra J, Frey M, Stebbins G, Pierre-Louis S, Iriarte J. Psychiatric and neurologic predictors of psychogenic pseudoseizure outcome. Neurology 1999;53:933-938.

11. Reuber M, Fernandez G, Bauer J, Singh DD, Elger CE. Interictal EEG abnormalities in patients with psychogenic nonepileptic seizures. Epilepsia 2002;43:1013-1020.

12. Kalogjera-Sackellares D, Sackellares JC. Impaired motor function in patients with psychogenic pseudoseizures. Epilepsia 2002;42:1600-1606.

13. Lempert T, Schmidt D. Natural history and outcome of psychogenic seizures: a clinical study in 50 patients. J Neurol 1990;237:35-38.

14. Pakalnis A, Drake ME, Jr., Phillips B. Neuropsychiatric aspects of psychogenic status epilepticus. Neurology 1991;41:11041106.

15. Walczak TS, Papacostas S, Williams DT, Scheuer ML, Lebowitz N, Notarfrancesco A. Outcome after diagnosis of psychogenic nonepileptic seizures. Epilepsia 1995;1131:1137.

16. Mokleby K, Blomhoff S, Malt UF, Dahlstrom A, Tauboll E, Gjerstad L. Psychiatric comorbidity and hostility in patients with psychogenic nonepileptic seizures compared with somatoform disorders and healthy controls. Epilepsia 2002;43:193-198.

17. Lesser RP, Lueders H, Dinner DS. Evidence for epilepsy is rare in patients with psychogenic seizures. Neurology 1983;33:502-504.

18. Lesser RP. Treating psychogenic nonepileptic seizures: easier said than done. Ann Neurol 2003;53:285-286.

19. Krumholz A, Niedermeyer E. Psychogenic seizures: a clinical study with follow-up data. Neurology 1983;33:498-502.

20. Kristensen O, Alving J. Pseudoseizures: risk factors and prognosis: a case-control study. Acta Neurol Scand 1992;85:177-180.

21. Ettinger AB, Devinsky O, Weisbrot DM, Ramakrishna RK, Goyal A. A comprehensive profile of clinical, psychiatric, and psychosocial characteristics of patients with psychogenic nonepileptic seizures. Epilepsia 1999;40(9):1292-1298.

22. Aboukasm A, Mahr G, Gahry BR, Thomas A, Barkley GL. Retrospective analysis of the effects of psychotherapeutic interventions on outcomes of psychogenic nonepileptic seizures. Epilepsia 1998;39:470-473.

23. Groves JE. Taking care of the hateful patient. N Engl J Med 1978;298:883-887. 